\section{Death of British space science?}

SIR - Ken Pounds, the chief executive of the UK Particle Physics and Astronomy Research Council (PPARC) responds (Nature 374, 491; 1995) to letters from the space astronomy community angry over a British withdrawal from the Integral space mission by shifting the blame for the present débâcle onto his predecessors at the Science and Engineering Research Council (SERC). A more important issue is what is done to resolve what is surely a matter of immense concern for a nation with a great heritage in space science.

Pounds says that "PPARC is actively working with its space partners to find additional funds for exploitation of future spaceflight opportunities". The PPARC policy (see Nature 372, 712; 1995) relies on the introduction of massive increases in efficiency to allow a 25 per cent reduction in member state contributions. The figure that would then be returned annually to the United Kingdom is that required to rebuild a domestic space budget which, as Pounds indicated, somehow disappeared in the final days of the SERC. I would like to see this policy buttressed by PPARC declaring that it will ring-fence any returned money for space. There must be a guarantee that if the massive changes targeted by the British are agreed, the domestic problem will not recur. PPARC has little to fear from such a step as I understand that it does have a guarantee from its masters at the Cabinet Office that money returned to PPARC from a reduced subscription to the European Space Agency (ESA) subscription will remain with PPARC.

Ironically, some of the efficiency gains promulgated by the British are already being implemented to get the Integral mission off the stocks without the United Kingdom. Large savings in payload cost (perhaps more than 15 per cent) are needed to keep Integral on track. The agency (and PPARC's partners in ESA) are being imaginative to achieve the necessary savings and they are certainly picking up on ideas put up by the United Kingdom.

Pounds agrees with his British critics that "it is vital that PPARC finds some way to ensure that the Integral débâcle does not recur with subsequent missions". I could not agree more. The British scientists looking to collaborate in Rosetta, the cornerstone cometary mission to be launched early in the next century, are already being treated with scepticism by their partners.

Could not PPARC improve its credibility by not only making its domestic policy consistent with what it has enunciated in ESA but also by being seen to make efforts domestically to bring its own house into order as a first step? Without UK credibility, its partners might be tempted by the option of proceeding to enjoy the benefits of efficiency brought by UK pressure but without coming near bailing out the United Kingdom.

None of this should get in the way of the fact that this year is a year of triumph for the ESA space science programme with the launch of six spacecraft, all of which have massive British involvement. The investments made, consequent on the fact that British space scientists have won most of their funding battles of the past decade, are just coming to fruition. If PPARC cannot find funds for Integral and Rosetta, the death knell for British space science may have sounded, but it is going to be a long and glorious death.

David Southwood

(Chairman, ESA Science Programme

Committee)

Blackett Laboratory,

Imperial College,

London SW7 2BZ, UK

\section{Diverging trends, intersecting issues}

SIR - The argument, presented in a leading article (Nature 374, 199-200; $1995)$ that the world should wait two, five or even more years before formulating a strict protocol on anthropogenic greenhouse gas emissions is an understandable one. But this does not mean that individual nations, or even groupings of nations such as the European Union, should away.

While it may well be prudent to call for a pause so as "to get the whole world on side", it is perhaps erroneous to suggest as you do in conclusion - that the reason is analogous to "unilateral disarmament being a fruitless policy". Unilateral interim action on anthropogenic climate change by those nations that can (primarily the developed nations) will do three things.

First, the developed (OECD) countries consume by far the most fossil fuels, both nationally and per capita, so that any progress in reducing emissions here has a good chance of lowering overall emissions in the short term. Second, moving to an economy less dependent on finite fossil fuels should provide those nations with greater security and potentially greater economic stability.

Third, in moving to an economy that is energy efficient and/or not based on fossil fuel, more appropriate technology will have been developed to a level where it might perhaps be used in countries yet to have made the transition. (There is a not set themselves their own goals straight fourth point in that an example will have been set: not one for environmental ethics - though that will be the case - but an example in practicality.)

So 'yes' to your call for caution at Berlin, but also 'yes' to unilateral action. Jonathan Cowie 5 Charlieville Road,

Northumberland Heath, Kent DA8 1HJ, UK

\section{Science and democracy}

SIR - You rightly highlight the need for public understanding of science (Nature 374, 291-292; 1995), but miss one of the most important reasons why it is desirable - democracy. It cannot be justified that, in nations that regard themselves as democratic, the population at large has little or no effective political control over the directions that research takes, and the uses to which scientific discoveries are put. Where we are concerned with the regulation of matters as diverse as embryo research, the use of transgenic plants in the field, or the safety (or otherwise) of nuclear power, it is surely in all our interests that as many as possible have knowledge that enables them to be involved in the decision-making process.

On the other hand, a properly informed and scientifically literate population would be able to enter into a rational discussion with the scientific community; this is surely the way in which the regulation of sensitive or controversial areas of research should be determined. The alternative is self-regulation by an unaccountable élite (a formula that has been singularly unsuccessful in other walks of life, for example the City of London), breeding resentment and alienation as a consequence.

The research community must not be a law unto itself; if it is seen to avoid giving the public the trust it deserves, it will in turn lose the trust of the public, and will deserve to do so.

\section{Robin Walters}

(Secretary, Scientists for Labour)

Department of Molecular Biology

and Biotechnology,

University of Sheffield,

Sheffield S102UH, UK

\section{Cold War against the Vatican}

An error was introduced in the editing of the letter from Serra and Neri (Nature 374, 589; 1995).

The last sentence of the first paragraph should have read: "Nothing could be further from the correctly interpreted facts of human reproduction and development than Godfrey's statement that ". . .we should envisage human persons coming into being . . . as each bright day is imperceptibly created during dawn."

NATURE • VOL 375 • 4 MAY 1995 